\title{
Accuracy of a nomogram for prediction of lymph-node metastasis detected with conventional histopathology and ultrastaging in endometrial cancer
}

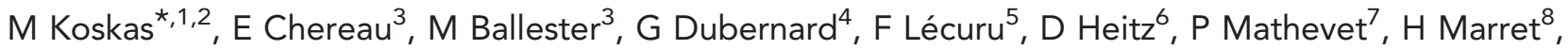 \\ D Querleu ${ }^{9}$, F Golfier ${ }^{10}$, E Leblanc ${ }^{11}$, D Luton ${ }^{1}$, R Rouzier ${ }^{12,13}$ and E Daraï ${ }^{2,13}$
}

${ }^{1}$ Department of Obstetrics and Gynaecology, Bichat University Hospital, Paris 75018, France; ${ }^{2}$ UMR S 938, CdR St Antoine UPMC University Paris 06, Paris, France; ${ }^{3}$ Department of Obstetrics and Gynaecology, Tenon University Hospital, Paris 75020, France; ${ }^{4}$ Department of Obstetrics and Gynaecology, Croix Rousse University Hospital, Lyon 69004, France; ${ }^{5}$ Department of Gynaecology, Georges Pompidou University Hospital, Paris 75015, France; ${ }^{6}$ Department of Gynaecology, Poissy-Saint Germain en Laye University Hospital, Poissy 78300, France; ${ }^{7}$ Department of Obstetrics and Gynaecology, Edouard Herriot University Hospital, Lyon 69003, France; ${ }^{8}$ Department of Obstetrics and Gynaecology, Bretonneau University Hospital, Tours 37044, France; ${ }^{9}$ Department of Surgical Oncology, Claudius Regaud Comprehensive Cancer Center, Toulouse 31300, France; ${ }^{10}$ Department of Obstetrics and Gynaecology, Centre Hospitalier Lyon Sud, Lyon 69230, France; ${ }^{11}$ Department of Surgical Oncology, Centre Oscar Lambret, Lille 59000, France and ${ }^{12}$ Department of Surgery, Institut Curie, Paris 75005, France

Background: We developed a nomogram based on five clinical and pathological characteristics to predict lymph-node (LN) metastasis with a high concordance probability in endometrial cancer. Sentinel LN (SLN) biopsy has been suggested as a compromise between systematic lymphadenectomy and no dissection in patients with low-risk endometrial cancer.

Methods: Patients with stage I-II endometrial cancer had pelvic SLN and systematic pelvic-node dissection. All LNs were histopathologically examined, and the SLNs were examined by immunohistochemistry. We compared the accuracy of the nomogram at predicting LN detected with conventional histopathology (macrometastasis) and ultrastaging procedure using SLN (micrometastasis).

Results: Thirty-eight of the 187 patients (20\%) had pelvic LN metastases, 20 had macrometastases and 18 had micrometastases. For the prediction of macrometastases, the nomogram showed good discrimination, with an area under the receiver operating characteristic curve (AUC) of 0.76, and was well calibrated (average error $=2.1 \%$ ). For the prediction of micro- and macrometastases, the nomogram showed poorer discrimination, with an AUC of 0.67, and was less well calibrated (average error $=10.9 \%)$.

Conclusion: Our nomogram is accurate at predicting LN macrometastases but less accurate at predicting micrometastases. Our results suggest that micrometastases are an 'intermediate state' between disease-free LN and macrometastasis.

${ }^{*}$ Correspondence: Dr M Koskas; E-mail: martin.koskas@bch.aphp.fr
${ }^{13}$ co-senior author.

Received 3 December 2012; revised 10 February 2013; accepted 12 February 2013; published online 12 March 2013

(c) 2013 Cancer Research UK. All rights reserved 0007-0920/13 
Endometrial cancer is the most common malignancy of the female genital tract and the seventh most common cause of cancer death in women in Western countries (Siegel et al, 2012). The histological type and grade of endometrial cancer and the depth of myometrial invasion are prognostic factors in early-stage disease as well as risk factors for lymph-node (LN) metastasis. However, the prognostic relevance of assessing LN status by lymphadenectomy is debated. Lymphadenectomy may be omitted in patients in low- and intermediate-risk groups, while pelvic and para-aortic lymphadenectomy is recommended in patients in high-risk groups, including patients with stage IB grade 3 endometrioid cancers and type II endometrial cancer. To provide evidence-based and individualised predictions, we developed a nomogram to predict LN status for endometrial cancer by combining selected clinical and pathological risk factors using a multivariate model (Bendifallah et al, 2012). This nomogram was based on five clinical and pathological characteristics to predict LN metastasis. It was developed using data from the Surveillance, Epidemiology and End Results (SEER) database and has been validated externally on a validation set. In both the training and the validation sets, a conventional histopathological examination was performed to assess LN metastases, and it is likely that only macrometastases were detected (Cote et al, 1999).

The results of a prospective multicenter study suggest that sentinel LN (SLN) biopsy could provide a trade-off between systematic lymphadenectomy and no dissection in patients with low-risk and intermediate-risk endometrial cancer (Ballester et al, 2011). Ultrastaging of LN, using serial sectioning and immunohistochemistry (IHC), is an important focus of the sentinel-node concept (Cote et al, 1999). In this study, almost half of patients with positive SLNs had occult metastases, including micrometastases and submicrometastases, which were not detected by conventional histology. The impact of micrometastases on the prognosis and the risk of recurrence has been shown in breast cancer (International (Ludwig) Breast Cancer Study Group, 1990; Cote et al, 1999). A case-control study in women with endometrial cancer showed that removal of micrometastases was associated with a significant increase in disease-free survival (Yabushita et al, 2001). Predictive factors of micrometastasis are unknown. Therefore, the predictive ability of a nomogram is also lacking. Additionally, such data could potentially be used as a surrogate to demonstrate the relative staging information provided by ultrastaging and micrometastasis.

The primary aim of this study was to evaluate and compare the accuracy of a nomogram when ultrastaging is performed on SLN in terms of calibration and discrimination. The secondary objective was to compare the values and distribution of nomogram scores in cases of LN micrometastasis and LN macrometastasis and in the absence of LN metastasis.

\section{MATERIALS AND METHODS}

Patients. From 2007 to 2011, 187 women with presumed stage III endometrial cancer determined using the 2009 FIGO (International Federation of Gynecology and Obstetrics) classification underwent hysterectomy with bilateral salpingooophorectomy, pelvic SLN and systematic pelvic-node dissection at four French centres. All the women had biopsy-proven endometrial cancer.

All women gave informed written consent to the therapeutic procedures and to the analysis of data related to their malignancy in accordance with institutional guidelines and the Declaration of Helsinki. The protocol was approved by the national ethics committee (CEROG 2012-GYN-10-01).
Medical records were reviewed to determine age, tumour stage, histology, surgical procedure, and the final pelvic and para-aortic node status. Outcome was obtained from the outpatient records.

Sentinel-node procedure. The SLN procedure was performed for each patient. The pelvic and lower para-aortic regions were carefully inspected by laparoscopy for lymph ducts and dye uptake by LN. All blue and/or hot LNs were removed separately. After the SLN procedure, systematic pelvic transperitoneal LN dissection extending from the external iliac (and obturator nerve) to the iliac bifurcation was performed. The absence of residual pelvic or paraaortic radioactivity was verified after pelvic lymphadenectomy. In accordance with French guidelines (Querleu et al, 2011), a paraaortic lymphadenectomy was recommended if metastases were detected on intraoperative histology or after definitive histology. Systematic pelvic and para-aortic lymphadenectomy was recommended for patients with type 2 endometrial cancer (clear-cell, serous endometrial cancer and carcinosarcoma).

\section{Histological analysis}

Conventional analysis of LN. Non-SLNs were sectioned at 3-mm intervals and submitted for paraffin embedding. Each block was sectioned at one to three levels, depending on the size of the tissue in the block, and stained with haematoxylin and eosin (H\&E). The size of LN metastases was estimated with an eyepiece micrometre. A macrometastasis was defined as a single focus of metastatic disease per LN, measuring $>2 \mathrm{~mm}$.

SLN-based ultrastaging. Ultrastaging was done for SLNs only, in accordance with a study validating the histological concept of the SLN procedure in endometrial cancer (Delpech et al, 2007). Each half-SLN was sectioned at 3-mm intervals. Each $3-\mathrm{mm}$ section was analysed at four additional levels of $150 \mu \mathrm{m}$. SLNs were examined by IHC with an anti-cytokeratin antibody cocktail (cytokeratins AE1-AE3; Dako Corporation, Glostrup, Denmark). Micrometastasis was defined as a focus of metastatic disease $<2 \mathrm{~mm}$. A submicrometastasis was defined as a single focus of metastatic disease measuring $<0.2 \mathrm{~mm}$, including the presence of single noncohesive tumour cells.

Nomogram evaluation. Recently, Bendifallah et al, 2012 reported a nomogram to predict LN invasion in early stages of endometrial cancer. The parameters included in this nomogram were age, race, histological type (type I: endometrioid carcinoma; type II: carcinosarcoma, clear-cell or papillary serous), tumour grade and primary tumour extension (endometrium, $<$ or $\geqslant 50 \%$ myometrial invasion or cervical stroma invasion). The mathematical model was developed using data from the SEER database, and the nomogram was validated on a single database that recorded patient data from four institutions.

For each patient, we calculated the score of the nomogram previously described and the probability of LN metastasis. We evaluated performance of the nomogram to predict nodal status assessed by conventional analysis of nodes and by ultrastaging.

Statistical analysis. Data were analysed and compared using the $\chi^{2}$ test, the Fisher's Exact test and the Kruskal-Wallis test.

The nomogram performance was quantified with respect to discrimination and calibration. Discrimination (i.e., whether the relative ranking of individual predictions was in the correct order) was quantified with the area under (AUC) the receiver operating characteristic curve (i.e., the agreement between observed outcome frequencies and predicted probabilities), and calibration was studied with graphical representations of the relationship between the observed outcome frequencies and the predicted probabilities (calibration curves). To quantify miscalibration we used the unreliability index $U$, which is the difference in $-2 \log$ likelihood of a model with both $\alpha$ and $\beta$ as free parameters and a model with 
$\alpha=0$ and $\beta=1$ (Miller et al, 1993). We also evaluated the average error $\left(E_{\text {aver }}\right)$ between the predictions and the observations obtained from the calibration curves. The clinical significance of the calibration is high because it reflects the accuracy of the individual predictions.

\section{RESULTS}

Patient and surgical characteristics of the population. Patient and surgical characteristics are reported in Table 1. There were no differences between the groups regarding age, BMI or parity.

All 187 patients underwent pelvic lymphadenectomy with SLN biopsy. Most had a laparoscopy (78\%). SLNs were not detected in 22 patients. Thirty-eight of the 187 patients (20\%) had pelvic LN metastases, 20 had macrometastases detected by conventional histologic examination and 18 had micrometastases detected by ultrastaging procedures. Twenty-six of the 187 patients (14\%) underwent para-aortic lymphadenectomy. Six of the 187 patients (3\%) had associated para-aortic LN metastases. None had isolated para-aortic LN metastases. For the SLN procedure, there were no differences between the groups for the rate of SLN detected, the number of SLN present or the number of non-SLN removed.

We found a significant difference between the three groups for the rate of para-aortic lymphadenectomy $(P<0.0001)$ and the occurrence of positive para-aortic nodes $(P=0.02)$.

Pathological characteristics. There were no differences between the groups regarding histological type, and there was a predominance of endometrioid carcinoma (88.8\%) (Table 2). Comparing patients with or without LN metastasis, we observed significant differences for the final grade $(P=0.0001)$ and the number of patients with lymphovascular scape invasion $(P<0.0001)$.

\begin{tabular}{|c|c|c|c|c|}
\hline & $\begin{array}{c}\text { Patients } \\
\text { without } \\
\text { LN } \\
\text { invasion } \\
(N=149)\end{array}$ & $\begin{array}{c}\text { Patients } \\
\text { with LN } \\
\text { micro- } \\
\text { metastases } \\
(N=18)\end{array}$ & $\begin{array}{c}\text { Patients } \\
\text { with } \\
\text { LN } \\
\text { macro- } \\
\text { metastases } \\
(\mathbf{N}=20)\end{array}$ & $\boldsymbol{P}$ \\
\hline Age (years) & $64.8 \pm 9.7$ & $68.0 \pm 10.5$ & $64.1 \pm 5.9$ & 0.57 \\
\hline $\mathrm{BMI}\left(\mathrm{kg} \mathrm{m}^{-2}\right)$ & $27.5 \pm 10.3$ & $26.0 \pm 8.4$ & $26.9 \pm 9.8$ & 0.73 \\
\hline Parity & $1.8 \pm 1.4$ & $2.1 \pm 1.3$ & $1.2 \pm 1.2$ & 0.09 \\
\hline SLN detected & $131(88 \%)$ & $18(100 \%)$ & $16(80 \%)$ & 0.16 \\
\hline $\begin{array}{l}\text { Number of SLNs } \\
\text { detected }\end{array}$ & $2.3 \pm 1.7$ & $2.9 \pm 1.7$ & $2.8 \pm 0.4$ & 0.13 \\
\hline $\begin{array}{l}\text { Number of } \\
\text { non-SLNs removed }\end{array}$ & $11.5 \pm 5.5$ & $12.8 \pm 7.7$ & $13.7 \pm 9.4$ & 0.82 \\
\hline \multicolumn{5}{|l|}{ Surgical route } \\
\hline $\begin{array}{l}\text { Laparoscopy } \\
\text { Open surgery and } \\
\text { laparoconversion }\end{array}$ & $\begin{array}{r}117(79 \%) \\
32(21 \%)\end{array}$ & $\begin{array}{r}16(89 \%) \\
2(11 \%)\end{array}$ & $\begin{array}{r}12(60 \%) \\
8(40 \%)\end{array}$ & 0.09 \\
\hline $\begin{array}{l}\text { Para-aortic } \\
\text { lymphadenectomy }\end{array}$ & $11(7 \%)$ & $3(17 \%)$ & $12(60 \%)$ & $<0.001$ \\
\hline $\begin{array}{l}\text { Number of para- } \\
\text { aortic LNs removed }\end{array}$ & $12.5 \pm 4.0$ & $16.7 \pm 6.8$ & $12.5 \pm 7.7$ & 0.42 \\
\hline $\begin{array}{l}\text { Positive para-aortic } \\
\text { lymphadenectomy }\end{array}$ & 0 & 0 & $6(30.0 \%)$ & 0.02 \\
\hline
\end{tabular}

The primary tumour invasion fell short of reaching statistical significance $(P=0.05)$, but the tumour size was significantly larger in patients with micro- or macrometastases $(P=0.02)$.

Accuracy of the nomogram to predict macrometastasis detected by conventional histologic analysis. We performed discrimination and calibration analysis according to LN status and method of detection, that is, macrometastasis detected by conventional histologic analysis, and macrometastasis and micrometastases detected by ultrastaging.

For the prediction of metastases detected by conventional histopathology, the nomogram showed good discrimination with an AUC of 0.76 (95\% CI 0.71-0.81) (Figure 1) and was well calibrated (average error $=2.1 \%$, maximal error $=10.5 \%$ ), with no statistical difference between predicted probabilities and observed proportions $(P$-value of the $U$ index $=0.56)($ Figure 2$)$.

Accuracy of the nomogram to predict nodal status including SLN-based ultrastaging. Considering LN metastasis detected by conventional histologic analysis and ultrastaging, the nomogram showed poorer discrimination, with an AUC of 0.67 (95\% CI 0.63-0.72) (Figure 1), and was less well calibrated (average error $=10.9 \%$, maximal error $=15.9 \%$ ) with a statistical difference between predictions and observations ( $P$-value of the $U$ index $<0.0001$ ) (Figure 2).

Impact of SLN-based ultrastaging. For the whole population, ultrastaging detected $10 \%$ additional metastasis. All these metastases were micrometasatasis. We studied the impact of SLN-based ultrastaging according to the risk of metastasis calculated by the nomogram (Table 3). The impact of ultrastadification was independent of the baseline risk: $9 \%, 8 \%, 11 \%$ and $11 \%$ in the four quartiles, respectively $(P=0.97)$.

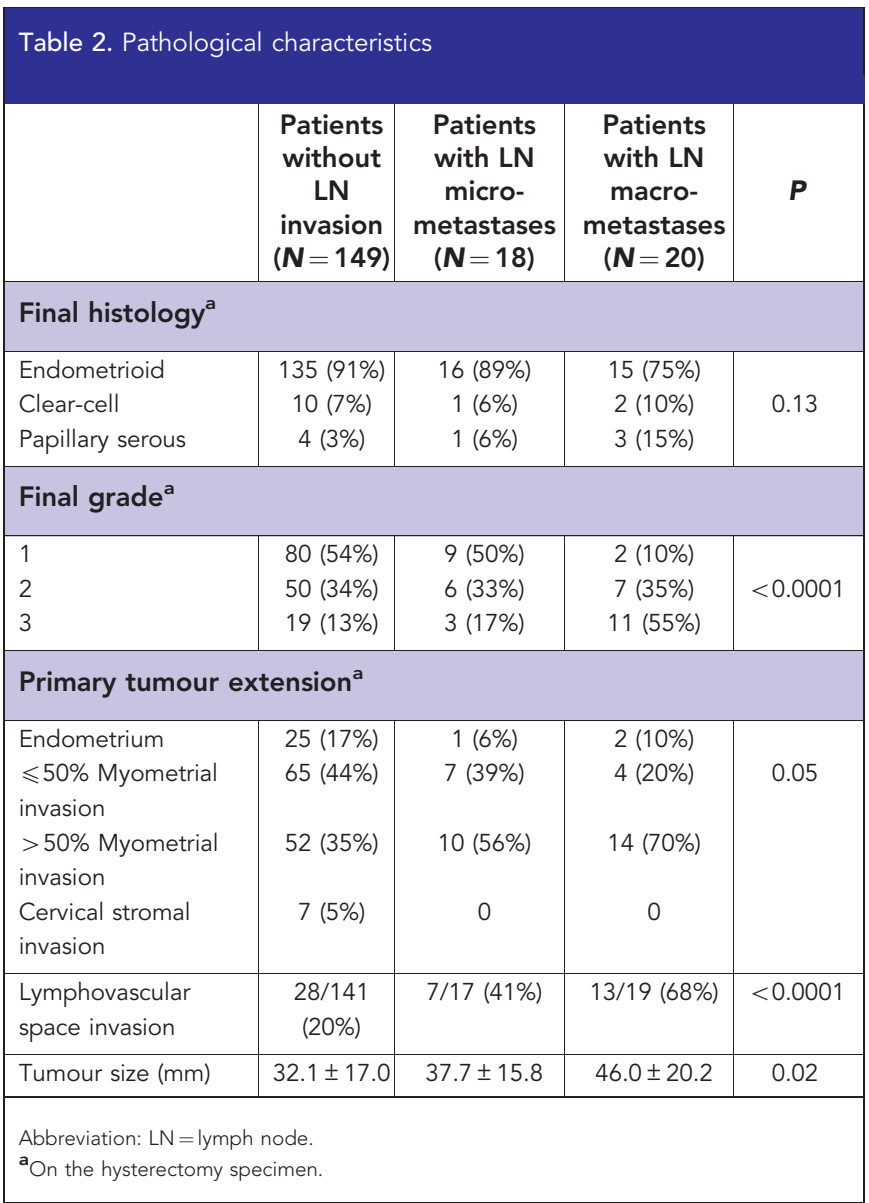




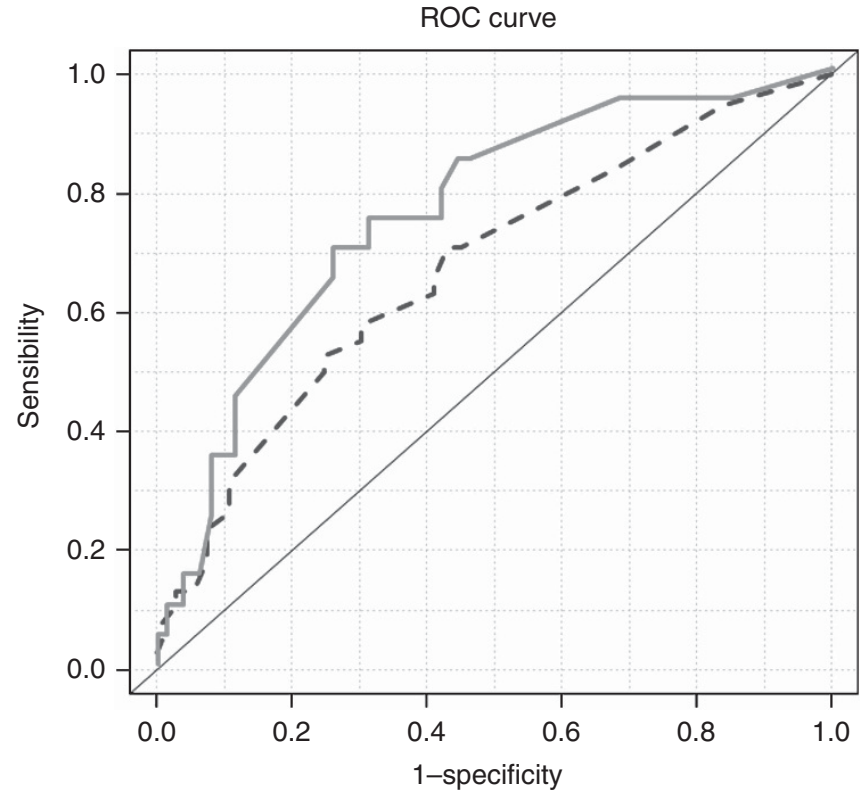

Figure 1. Receiver operating characteristic curves of the model. Solid line (red): Receiver operating characteristic curves of the nomogram to predict macro LN metastases. AUC $=0.76$ ( $95 \% \mathrm{Cl} 0.71-0.81$ ). Dotted line (blue): Receiver operating characteristic curves of the nomogram to predict micro and macro LN metastases. AUC $=0.67(95 \% \mathrm{Cl} 0.63-$ 0.72). The color reproduction of this figure is available on the British Journal of Cancer online.

\section{DISCUSSION}

Although micrometastases in breast cancers have been described previously, the concept of micrometastases in endometrial cancer has only recently been reported and investigated. The present study is the largest to focus on the primary tumoral characteristics in patients with micrometastasis. To better comprehend micrometastasis, we evaluated the accuracy of a nomogram designed to predict LN invasion in endometrial cancer. For the prediction of macrometastases, the nomogram showed good discrimination and was well calibrated, but for the prediction of LN metastasis detected by ultrastaging, the nomogram showed worse discrimination and was less well calibrated. The frequency of micrometastasis remained stable irrespective of the probability of LN metastasis calculated through the nomogram.

For patients with early-stage endometrial cancer, two randomized trials have shown that pelvic lymphadenectomy has no effect on overall or recurrence-free survival, and leads to a higher incidence of early and late complications (Benedetti Panici et al, 2008; Kitchener et al, 2009). In the ASTEC study (Kitchener et al, 2009), the risk of developing short-term major surgical complications was low in both groups, but more women in the lymphadenectomy group than in the standard surgery group developed specific complications. Moreover, more patients required blood transfusion in the lymphadenectomy group and the median length of operation was higher when lymphadenectomy was performed. Additionally, after adjuvant treatment, more women in the lymphadenectomy group than in the standard surgery group reported moderate or severe morbidity or treatmentrelated complications. Similarly, in the experience of Benedetti Panici et al, 2008, both early and late postoperative complications occurred significantly more frequently in patients who had received pelvic systematic lymphadenectomy. In accordance with the findings of these two prospective studies showing that

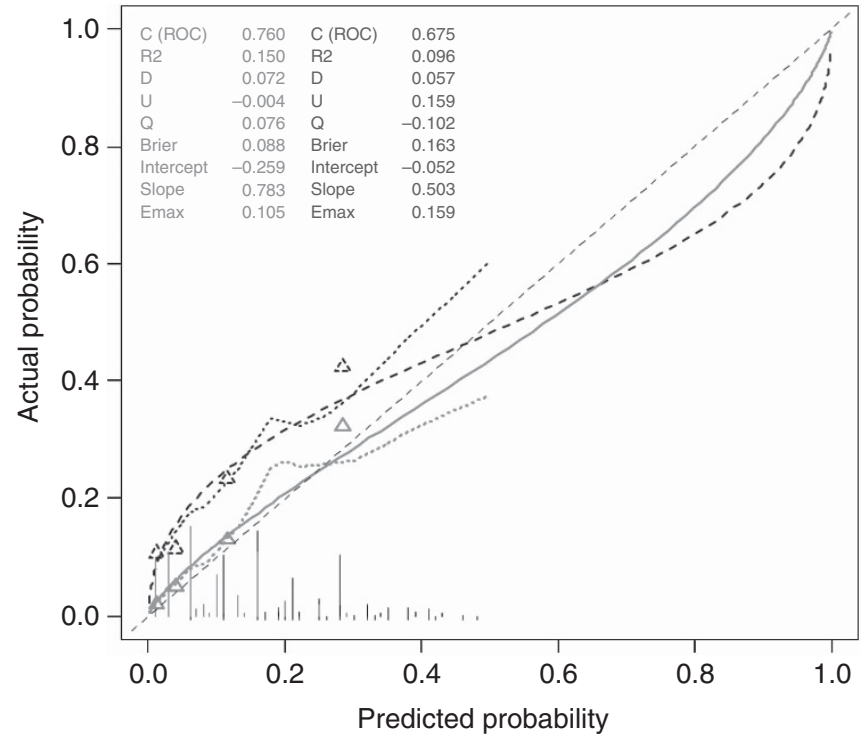

Figure 2. Calibration of the nomogram to predict metastatic lymph node involvement. The horizontal axis represents the predicted probability of metastatic lymph nodes, and the vertical axis represents the actual probability of metastatic lymph nodes. Perfect prediction would correspond to the $45^{\circ}$ broken line. Solid line (red): Calibration of the nomogram to predict macrometastatic LN involvement. The horizontal axis represents the predicted probability of macrometastatic LNs, and the vertical axis represents the actual probability of macrometastatic LNs. Perfect prediction would correspond to the $45^{\circ}$ dashed line. Average error $=2.1 \%$, maximal error $=10.5 \%$. Dotted line (blue): Calibration of the nomogram to predict micro- and macrometastatic LN involvement. The horizontal axis represents the predicted probability of micro- and macrometastatic LNs, and the vertical axis represents the actual probability of micro- and macrometastatic LNs. Average error $=10.9 \%$, maximal error $=15.9 \%$. The color reproduction of this figure is available on the British Journal of Cancer online.

lymphadenectomy is not such a low morbidity procedure, we believe that an alternative to systematic pelvic lymphadenectomy in early endometrial cancer (provided by SLN or the use of a nomogram) should be considered, especially as the benefit of lymphadenectomy has not been proven.

In comparison with other studies reporting $\mathrm{LN}$ invasion in presumed stage I and II endometrial cancer, the rate of nearly $21 \%$ appears to be high. This difference could be explained by the fact that most other studies did not include micrometastases. For example, in the two major randomised trials concerning lymphadenectomy in endometrial cancer (Benedetti Panici et al, 2008; Kitchener et al, 2009), the rates of patients with pelvic LN were 9\% (Kitchener et al, 2009) and 13\% (Benedetti Panici et al, 2008), respectively. These rates of LN metastases were most likely underestimated. In the present study, IHC detected metastases that would have not been diagnosed with conventional histology in 18 of 187 patients $(9.6 \%)$ with detected SLNs, representing 18 of 38 patients $(47.4 \%)$ with metastases.

In the present study, the rate of micrometastasis is higher than in other reports (Gonzalez Bosquet et al, 2003; Pelosi et al, 2003; Niikura et al, 2007; McCoy et al, 2012). The difference is most likely explained by the methodology of screening for micrometastases. In other reports, SLN was not performed, and the whole lymphadenectomy specimen was examined for micrometastasis. For example, in McCoy's experience of screening for micrometastasis in 51 patients, only $151 \mathrm{LN}$ paraffin blocks were obtained, whereas the mean number of LNs removed per patient 


\begin{tabular}{l} 
Table 3. Actual probability of LN micro- and macrometastasis according to the probability of LN metastasis given by the nomogram \\
\begin{tabular}{|l|c|c|c|c|c|}
\hline & Whole population & 1st quartile & 2nd quartile & 3rd quartile & 4th quartile \\
\hline Predicted probability of LN metastasis & $0.5-50 \%$ & $\leqslant 2 \%$ & $3-4 \%$ & $5-14 \%$ & $>$ or $=15 \%$ \\
\hline$N$ & 187 & 54 & 39 & 38 & 56 \\
\hline Mean predicted probability of LN metastasis & $9 \%$ & $1 \%$ & $4 \%$ & $9 \%$ & $22 \%$ \\
\hline Observed probability of LN macrometastasis & $20 / 187(11 \%)$ & $1 / 54(2 \%)$ & $2 / 39(5 \%)$ & $4 / 38(11 \%)$ & $13 / 56(23 \%)$ \\
\hline Observed probability of LN micrometastasis & $18 / 187(10 \%)$ & $5 / 54(9 \%)$ & $3 / 39(8 \%)$ & $4 / 38(11 \%)$ & $6 / 56(11 \%)$ \\
\hline Abbreviation: $L N=$ lymph node. & & & &
\end{tabular} \\
\hline
\end{tabular}

was 12 (McCoy et al, 2012). Because micrometastases are usually defined as positive staining of cells $<2 \mathrm{~mm}$ in the greatest dimension, searching for them in all LNs removed during pelvic lymphadenectomy is hardly feasible. In accordance with a study validating the histological concept of the SLN procedure, ultrastaging was usually only performed for SLNs (Delpech et al, 2007). Cytokeratin, a constituent of the cytoskeleton of normal and malignant epithelial cells, is usually only present in tumour metastasis (Lindemann et al, 1992). However, Gould et al (1995) reported that cytokeratin-positive interstitial reticulum cells were increased in non-neoplastic inflammatory lymphadenopathies as well as neoplastic lymphadenopathies. This suggests that cytokeratin expressed in regional LNs does not always originate in neoplastic epithelial cells. In order to identify the presence of occult metastases in LNs, Yabushita et al, 2001 examined cytokeratin expression in cells other than tumour cells in LNs and its predictive value for recurrence in early-stage endometrial carcinoma. Cytokeratin expression was observed in $16 \%$ of LNs with unconfirmed metastasis, which were obtained from 14 of 36 patients with Stage I disease. Five of 14 patients with LNs expressing cytokeratin had recurrent disease in the pelvic cavity, while all 22 patients with unconfirmed cytokeratin expression in their LNs showed no recurrence. Multivariate analysis identified cytokeratin expression as an independent risk factor for recurrence in Stage I endometrial cancer. We share the conclusions of these authors suggesting that the immunohistochemical expression of cytokeratinin LNs with undetected metastases could be associated with occult LNs metastases.

Using a nomogram, we tried to better characterise and to compare the primary tumoral characteristics in patients with micrometastases. The nomogram we developed to predict LN metastasis in endometrial cancer is mainly based on primary tumoral characteristics (cancer grade, histologic subtype, depth of invasion, cervical involvement) and the training set was extracted from patients included in the SEER database who were treated between 1988 and 2007. In this population study, even if it is not detailed, IHC was most likely not performed systematically because the current standard for detection of endometrial LN metastasis involves H\&E staining only. Our nomogram has been shown to be a good predictor of LN metastasis (Bendifallah et al, 2012). Interestingly, the nomogram approach permits the evaluation of the primary tumoral aggressiveness in each patient. Our results suggest that primary tumoral characteristics between patients without LN micrometastases and patients with macrometastases are significantly different. Interpretation of the frequency of micrometastases according to the nomogram probability suggests that compared with macrometastases, micrometastases may occur earlier and in tumours with lower carcinologic aggressiveness. These results suggest that micrometastasis could be an intermediate state' between disease-free LN and macrometastasis. The initial study (Bendifallah et al, 2012) was conducted in order to develop and evaluate a nomogram to predict the LN metastatic risk for patients with presumed stage I and II endometrial cancer. In the original study, we chose not to propose a cutoff value, as the use of a nomogram permits to decide whether secondary lymphadenectomy is suitable at an individual level. However, we could investigate if a threshold could be established by focusing on the negative predictive value to build a model that discriminates a low-risk group for nodal metastasis. We believe that the most important goal for developing this model would be to obtain the best negative predictive value in order to prevent misclassifying a patient with LN metastasis.

In the present study few patients underwent para-aortic lymphadenectomy and because of comorbidities para-aortic lymphadenectomy could be omitted. We know that the expected rate of para-aortic LN mestastasis in patients with metastatic pelvic LNs can reach 30-50\% (Bristow et al, 2003; Mariani et al, 2004), which is similar to our finding that 6 of the 12 patients with pelvic LN macrometastasis who underwent para-aortic lymphadenectomy had also aortic involvement. However, it is possible that we 'missed' isolated para-aortic LN metastases but the number would be very low. Two methods can be used to assess this number:

-When considering the results of the ASTEC study (Kitchener et al, 2009), 6\% of patients with positive LN metastasis had paraaortic involvement. If we transpose the results of the ASTEC study to our population sample, as 20 patients had macroscopic pelvic LN metastases, one to two patient(s) could have had isolated paraaortic LN metastases.

-In the experiment by Mariani et al (2004) focusing on paraaortic dissemination in endometrial cancer, only $2 \%$ of patients with negative pelvic LNs had para-aortic metastasis. As the findings of this study are in accordance with the others involving more than a 100 patients (Morrow et al, 1991; Ayhan et al, 1995; Hirahatake et al, 1997), we can apply this rate to our population sample. In doing so, we estimate the number of patients with isolated para-aortic LN metastases to be three.

Considering the low number of patients with isolated paraaortic LN metastases (one to three patients) in comparison with the number of patients with pelvic para-aortic LN metastases, it is unlikely that this would affect the discriminative accuracy of the nomogram to a large extent. Moreover, in the SEPAL study (Todo et al 2010), the incidence of para-aortic LN involvement in intermediate- and high-risk groups is only $14 \%$ and $18 \%$, suggesting that the majority of the patients has little benefice of systematic para-aortic lymphadenectomy.

The impact of micrometastases remains debatable. Despite a favourable prognosis in patients with negative LN, up to $15 \%$ of patients experience a recurrence of disease. One hypothesis for this recurrence is the false-negative rates with current pathologic LN assessments. Interestingly, this risk of occult metastasis is independent of the baseline risk of nodal metastasis. Authors have investigated numerous ways to improve detection of LN metastasis, such as serial sectioning (Reich et al, 1996), IHC and molecular testing, both alone or in combination (Bezu et al, 2010). Using these combination techniques in FIGO stage I-IV cancers demonstrated a detection rate of micrometastases between 0 and 
$20 \%$ (Bezu et al, 2010). Unfortunately, the impact of LN metastasis detected by ultrastaging has only been investigated through small case-control studies involving 50 patients or less (Yabushita et al, 2001; Gonzalez Bosquet et al, 2003; McCoy et al, 2012). The results of these studies provide contradictory conclusions on the prognostic significance of micrometastasis.

Without information on LN status, indications for adjuvant therapy are based on uterine features alone. Omitting lymphadenectomy carries a risk of inadequate staging, leading to secondary lymphadenectomy or systematic adjuvant radiotherapy. We believe SLN biopsy is a good alternative for the subgroup of patients who will not benefit from complete lymphadenectomy, which is associated with increased morbidity.

In conclusion, we founded that prediction of LN metastasis detected by conventional histopathology (macrometastasis) is feasible using a nomogram based on pathological characteristics of the hysterectomy specimen, whereas prediction of LN metastasis detected by ultrastaging on SLN (micrometastasis) is not correlated with LN involvement probability. Consequently, our results suggest that SLN biopsy should be performed for low and intermediaterisk endometrial cancers if we consider that this information modifies prognostic and adjuvant therapy. However, the prognostic significance of micrometastases needs to be evaluated more extensively in endometrial cancer.

\section{CONFLICT OF INTEREST}

The authors declare no conflict of interest.

\section{REFERENCES}

Ayhan A, Tuncer ZS, Tuncer R, Yuce K, Kucukali T (1995) Tumor status of lymph nodes in early endometrial cancer in relation to lymph node size. Eur J Obstet Gynecol Reprod Biol 60(1): 61-63.

Ballester M, Dubernard G, Lecuru F, Heitz D, Mathevet P, Marret H, Querleu D, Golfier F, Leblanc E, Rouzier R, Darai E (2011) Detection rate and diagnostic accuracy of sentinel-node biopsy in early stage endometrial cancer: a prospective multicentre study (SENTI-ENDO). Lancet Oncol 12: 469-476.

Bendifallah S, Genin AS, Naoura I, Chabbert Buffet N, Clavel Chapelon F, Haddad B, Luton D, Darai E, Rouzier R, Koskas M (2012) A nomogram for predicting lymph node metastasis of presumed stage I and II endometrial cancer. Am J Obstet Gynecol 207: e1-8.

Benedetti Panici P, Basile S, Maneschi F, Alberto Lissoni A, Signorelli M, Scambia G, Angioli R, Tateo S, Mangili G, Katsaros D, Garozzo G, Campagnutta E, Donadello N, Greggi S, Melpignano M, Raspagliesi F, Ragni N, Cormio G, Grassi R, Franchi M, Giannarelli D, Fossati R, Torri V, Amoroso M, Croce C, Mangioni C (2008) Systematic pelvic lymphadenectomy vs. no lymphadenectomy in early-stage endometrial carcinoma: randomized clinical trial. J Natl Cancer Inst 100: 1707-1716.

Bezu C, Coutant C, Ballester M, Feron JG, Rouzier R, Uzan S, Darai E (2010) Ultrastaging of lymph node in uterine cancers. J Exp Clin Cancer Res 29: 5.

Bristow RE, Zahurak ML, Alexander CJ, Zellars RC, Montz FJ (2003) FIGO stage IIIC endometrial carcinoma: resection of macroscopic nodal disease and other determinants of survival. Int J Gynecol Cancer 13: 664-672.

Cote RJ, Peterson HF, Chaiwun B, Gelber RD, Goldhirsch A, CastiglioneGertsch M, Gusterson B, Neville AM (1999) Role of immunohistochemical detection of lymph-node metastases in management of breast cancer. International Breast Cancer Study Group. Lancet 354: 896-900.

Delpech Y, Cortez A, Coutant C, Callard P, Uzan S, Darai E, Barranger E (2007) The sentinel node concept in endometrial cancer: histopathologic validation by serial section and immunohistochemistry. Ann Oncol 18: 1799-1803.
Gonzalez Bosquet J, Keeney GL, Mariani A, Webb MJ, Cliby WA (2003) Cytokeratin staining of resected lymph nodes may improve the sensitivity of surgical staging for endometrial cancer. Gynecol Oncol 91: 518-525.

Gould VE, Bloom KJ, Franke WW, Warren WH, Moll R (1995) Increased numbers of cytokeratin-positive interstitial reticulum cells (CIRC) in reactive, inflammatory and neoplastic lymphadenopathies: hyperplasia or induced expression? Virchows Arch 425: 617-629.

Hirahatake K, Hareyama H, Sakuragi N, Nishiya M, Makinoda S, Fujimoto S (1997) A clinical and pathologic study on para-aortic lymph node metastasis in endometrial carcinoma. J Surg Oncol 65: 82-87.

International (Ludwig) Breast Cancer Study Group (1990) Prognostic importance of occult axillary lymph node micrometastases from breast cancers. Lancet 335(8705): 1565-1568.

Kitchener H, Swart AM, Qian Q, Amos C, Parmar MK (2009) Efficacy of systematic pelvic lymphadenectomy in endometrial cancer (MRC ASTEC trial): a randomised study. Lancet 373: 125-136.

Lindemann F, Schlimok G, Dirschedl P, Witte J, Riethmuller G (1992) Prognostic significance of micrometastatic tumour cells in bone marrow of colorectal cancer patients. Lancet 340: 685-689.

Mariani A, Keeney GL, Aletti G, Webb MJ, Haddock MG, Podratz KC (2004) Endometrial carcinoma: paraaortic dissemination. Gynecol Oncol 92: 833-838.

McCoy A, Finan MA, Boudreaux FT, Tucker JA, Lazarchick JJ, Donnell RM, Rocconi RP (2012) The incidence and clinical significance of lymph node micrometastases determined by immunohistochemical staining in stage I-lymph node negative endometrial cancer. Histol Histopathol 27: 181-185.

Miller ME, Langefeld CD, Tierney WM, Hui SL, McDonald CJ (1993) Validation of probabilistic predictions. Med Decis Making 13: 49-58.

Morrow CP, Bundy BN, Kurman RJ, Creasman WT, Heller P, Homesley HD, Graham JE (1991) Relationship between surgicalpathological risk factors and outcome in clinical stage I and II carcinoma of the endometrium: a Gynecologic Oncology Group study. Gynecol Oncol 40: $55-65$.

Niikura H, Okamoto S, Yoshinaga K, Nagase S, Takano T, Ito K, Yaegashi N (2007) Detection of micrometastases in the sentinel lymph nodes of patients with endometrial cancer. Gynecol Oncol 105: 683-686.

Pelosi E, Arena V, Baudino B, Bello M, Giusti M, Gargiulo T, Palladin D, Bisi G (2003) Pre-operative lymphatic mapping and intra-operative sentinel lymph node detection in early stage endometrial cancer. Nucl Med Commun 24: 971-975.

Querleu D, Planchamp F, Narducci F, Morice P, Joly F, Genestie C, Haie-Meder C, Thomas L, Quenel-Tueux N, Darai E, Dorangeon PH, Marret H, Taieb S, Mazeau-Woynar V (2011) Clinical practice guidelines for the management of patients with endometrial cancer in France: recommendations of the Institut National du Cancer and the Societe Francaise d'Oncologie Gynecologique. Int J Gynecol Cancer 21: 945-950.

Reich O, Winter R, Pickel H, Tamussino K, Haas J, Petru E (1996) Does the size of pelvic lymph nodes predict metastatic involvement in patients with endometrial cancer? Int J Gynecol Cancer 6: 445-447.

Siegel R, Naishadham D, Jemal A (2012) Cancer statistics, 2012. CA Cancer J Clin 62: 10-29.

Todo Y, Kato H, Kaneuchi M, Watari H, Takeda M, Sakuragi N (2010) Survival effect of para-aortic lymphadenectomy in endometrial cancer (SEPAL study): a retrospective cohort analysis. Lancet 375: 1165-1172.

Yabushita H, Shimazu M, Yamada H, Sawaguchi K, Noguchi M, Nakanishi M, Kawai M (2001) Occult lymph node metastases detected by cytokeratin immunohistochemistry predict recurrence in node-negative endometrial cancer. Gynecol Oncol 80: 139-144.

This work is published under the standard license to publish agreement. After 12 months the work will become freely available and the license terms will switch to a Creative Commons AttributionNonCommercial-Share Alike 3.0 Unported License. 